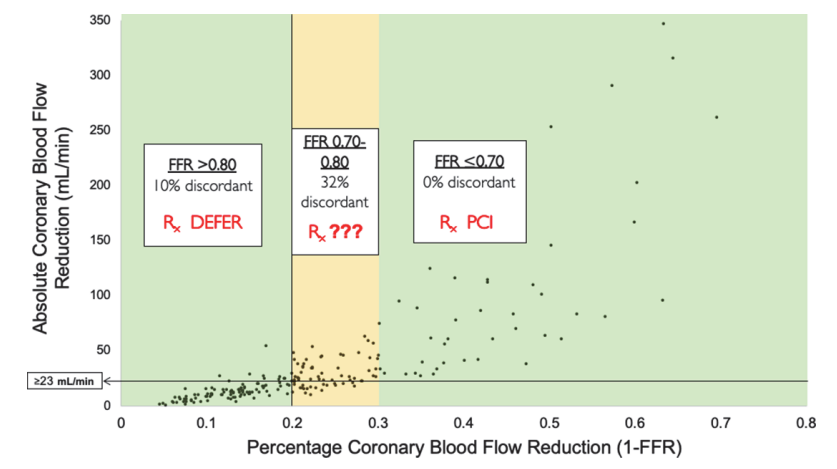

Abstract 33 Figure 1

increased agreement to near perfect $(\kappa=0.90)$ and required aCBF to be measured in $32 \%$ of cases. CMVR was higher in females at baseline and hyperaemia ( 1.59 vs 1.21 and 0.86 vs $0.68 \mathrm{mmHg} \cdot \mathrm{mL} \cdot \mathrm{min}-1$, respectively, $\mathrm{P}<0.05)$.

Conclusions In the largest study of aCBF to date, $19.5 \%$ of cases identified as physiologically significant by FFR were identified as non-significant by aCBF reduction criteria. Pressure-flow discordance was associated with variability in CMVR and may explain why around 20\% of patients experience persistent angina following FFR-guided PCI. Combined FFR + aCBF assessment may be valuable, particularly in the FFR grey zone. virtuQ ${ }^{\mathrm{TM}}$ may have a complementary role in selecting patients for PCI and help diagnose microvascular disease.

Conflict of Interest None

\section{NEXT GENERATION P2Y12 INHIBITORS IMPROVE SURVIVAL IN ACS: AN ANALYSIS FROM THE BRITISH CARDIOVASCULAR INTERVENTION SOCIETY DATABASE}

${ }^{1}$ Fahmida Mannan, ${ }^{1}$ Sushant Saluja, ${ }^{2}$ Hussain Contractor, ${ }^{1}$ Nik Abidin, ${ }^{1}$ Alan Fitchet, ${ }^{1}$ Lwin Tin, 'Peter Woolfson, ${ }^{3}$ Scot Garg, ${ }^{4}$ Simon Anderson. 'Salford Royal NHS Foundation Trust, Salford, UK; ${ }^{2}$ University Hospital of South Manchester, NHS Foundation Trust, NorthWest Heart Centre, Manchester, UK; ${ }^{3}$ Royal Blackburn Hospital, East Lancashire Hospital NHS Trust, Blackburn, UK; ${ }^{4}$ University of West Indies, Cavehill Campus, Barbados

\subsection{6/heartjnl-2021-BCS.34}

Background Dual antiplatelet therapy (DAPT) is the standard care following presentation with an acute coronary syndrome (ACS), but there remains debate regarding the relative benefits of the available P2Y12 receptor antagonists and their optimal combination with aspirin, particularly in those treated with percutaneous coronary intervention (PCI).

Methods We performed a retrospective analysis of all PCI procedures undertaken in patients with ACS recorded in the British Cardiovascular Intervention Society (BCIS) database between 2007 and 2014 who were treated with DAPT consisting of aspirin and one of either clopidogrel, prasugrel or ticagrelor. The primary outcome measure was 30-day all-cause mortality, with secondary outcome measures of mortality at 1 and 5 years. Odds ratios (OR) for mortality were determined from multivariable logistic regression models allowing for clustering by hospital.

Results Among 259,255 eligible patients with 2 million person-years of observation, $7.4 \%(19,101)$ of patients had ticagrelor, $7.4 \%(\mathrm{n}=19,161)$ had prasugrel and $85.2 \%$ $(n=220,993)$ were treated with clopidogrel for ACS. A total of 41,107 (12.2\%) patients died during a median of follow-up of 3.2 years (IQR: 1.6-5.2 years). Crude mortality rates were 34.7 (clopidogrel), 30.6 (prasugrel), and 36.9 deaths per 1000-person-years for ticagrelor treated ACS. In an age-sex unadjusted multinomial logistic regression analysis, mortality rates at 1 year in those treated with aspirin and ticagrelor were 64\% lower [OR 0.34, 95\% CI (0.32-0.36)] than those receiving DAPT with clopidogrel. DAPT with prasugrel was associated with a $27 \%$ lower mortality compared to DAPT with clopidogrel (OR $0.73(0.69-0.77), \mathrm{p}<0.0001)$. Stratifying by ACS status, the age-sex adjusted 1-year mortality rate for ticagrelor compared with clopidogrel was $63 \%$ lower [(OR 0.37 $(0.34-0.40)$ ] in STEMI and $80 \%$ lower in NSTEMI [(OR $0.20(0.18-0.23), \mathrm{p}<0.0001)]$. The reduction in mortality at 1 year in the prasugrel versus clopidogrel group was relatively greater $(57 \%)$ in individuals with STEMI [(OR $0.43(0.40-0.45), \quad \mathrm{p}<0.0001)]$ compared to those with NSTEMI [(OR $0.64(0.55-0.74), \mathrm{p}<0.0001)]$.

Conclusions This very large, real-world dataset of patients presenting with ACS demonstrates a significant net clinical benefit favouring the use of ticagrelor and prasugrel over clopidogrel in ACS patients for DAPT. This analysis concurs with the data from the landmark TRITON and PLATO RCTs, suggesting these agents should be considered as the standard of care in the management of ACS.

Conflict of Interest None

\section{THE IMPACT OF CARDIOVASCULAR DISEASE ON SEX- SPECIFIC ADVERSE OUTCOMES FOLLOWING INTACT ABDOMINAL AORTIC ANEURYSM REPAIR: A SYSTEMATIC REVIEW, META-ANALYSIS \& META- REGRESSION}

${ }^{1}$ Anna Louise Pouncey, ${ }^{1}$ Michael David, ${ }^{2}$ Rachael Morris, ${ }^{1}$ Pinar Ulug, ${ }^{1}$ Guy Martin, ${ }^{1}$ Colin Bicknell, ${ }^{1}$ Janet Powell. ${ }^{1}$ Imperial College London, London, UK; ${ }^{2}$ Kings College London

\subsection{6/heartjnl-2021-BCS.35}

Introduction Cardiovascular disease is a major cause of death in men with an AAA. Women experience higher operative mortality than men for open (OAR) and endovascular (EVAR) repair of intact abdominal aortic aneurysm (AAA), but the reason for this is not yet established. This study aimed to define differences in cardiovascular pre-operative co-morbidity and peri/post-operative complications for men and women under-going OAR and EVAR, to explore the impact of cardiovascular disease on adverse outcomes following intact AAA repair.

Methods A systematic review, meta-analysis and meta-regression of sex-specific differences in mortality and complications was conducted and reported according to PRISMA and Cochrane guidance, and registered with Prospero (CRD42020176398). Papers reporting outcomes for men and women, following intact primary AAA repair, from 2000-2020 world-wide were included. Separate analyses were conducted for EVAR and OAR. Data sources included: Medline, Embase and CENTRAL databases 2005-2020 searched using ProQuest Dialog $^{\text {TM }}$. 
Results A total of 26 studies (371,215 men, 65,465 women) were included. Risk of 30-day mortality was higher in women for OAR and more so for EVAR (OR [95\%CI] 1.49 $[1.37,1.61]$ and $1.86[1.59,2.17]$ respectively), and remained following multivariate risk factor adjustment. Although assessment of pre-operative co-morbidities was limited by heterogeneity, cardiac disease was more commonly diagnosed in men (OAR OR 0.72 [0.59,0.88]; EVAR OR 0.65 [0.48,0.87]) no differences in peripheral vascular disease or smoking history were observed. However, following OAR, the likelihood of acute coronary complications was similar for both sexes (OR 1.18 [0.98-1.42]) and following EVAR, for women, the likelihood of acute coronary complication was significantly higher (OR 1.19 [1.03,1.37]). Renal injury, arterial injury and limb ischemia were also more common in women undergoing EVAR (ORs 1.46 [1.22-1.72], 3.02 [1.62-5.65], 2.13 [1.483.06] respectively) (figure 1). Meta-regression revealed cardiac complications were significantly associated with greater mortality risk differential between men and women (Figure 2); the association of renal complications with death was of borderline significance.
Conclusions The excess risk of 30-day mortality for women following AAA repair has not abated with time, with an increased risk differential for EVAR over OAR. Although our meta-analysis identified a lower prevalence in pre-operative diagnosis of coronary artery disease amongst women, acute coronary complications were significantly higher for women following EVAR, and similar to men following OAR. An increase in acute coronary complications for women compared to men was associated with a higher mortality risk differential. Women were also at greater risk of additional arterial complications leading to renal injury and limb ischaemia. These findings suggest that cardiovascular disease has significant impact on adverse outcomes for women after AAA repair. Further work to improve identification and treatment of cardiovascular disease in women is needed and has the potential to address disparity in outcomes for AAA repair. Figure 1. Comparison of 30-day complications for men and women following (a) OAR and (b) EVAR. Figure 2. (a) Meta-regression of $\log$ odds of (a) cardiac complications and (b) renal complications against log odds of 30-day/in-hospital mortality for women (vs. men) following endovascular repair

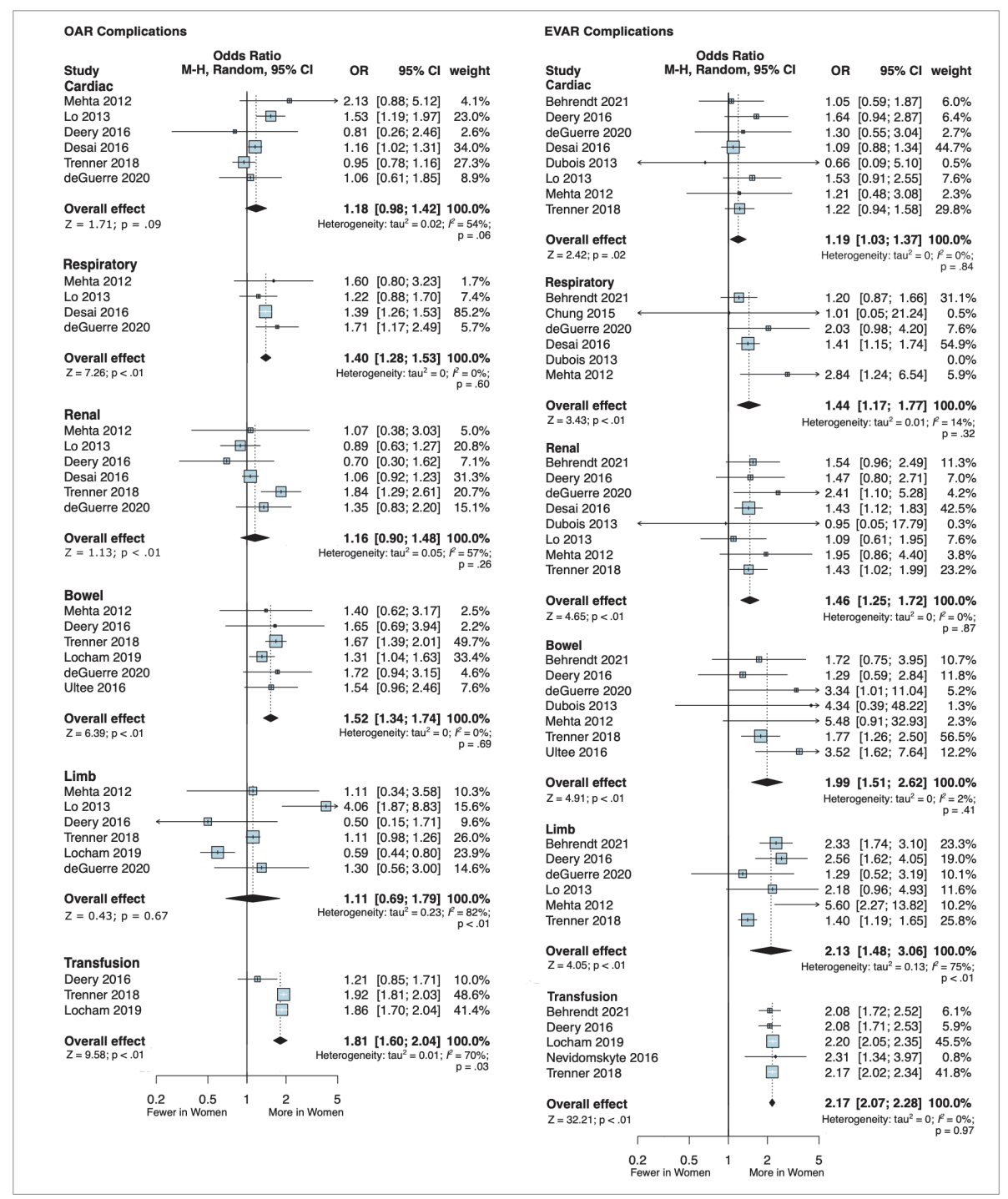

Abstract 35 Figure 1 


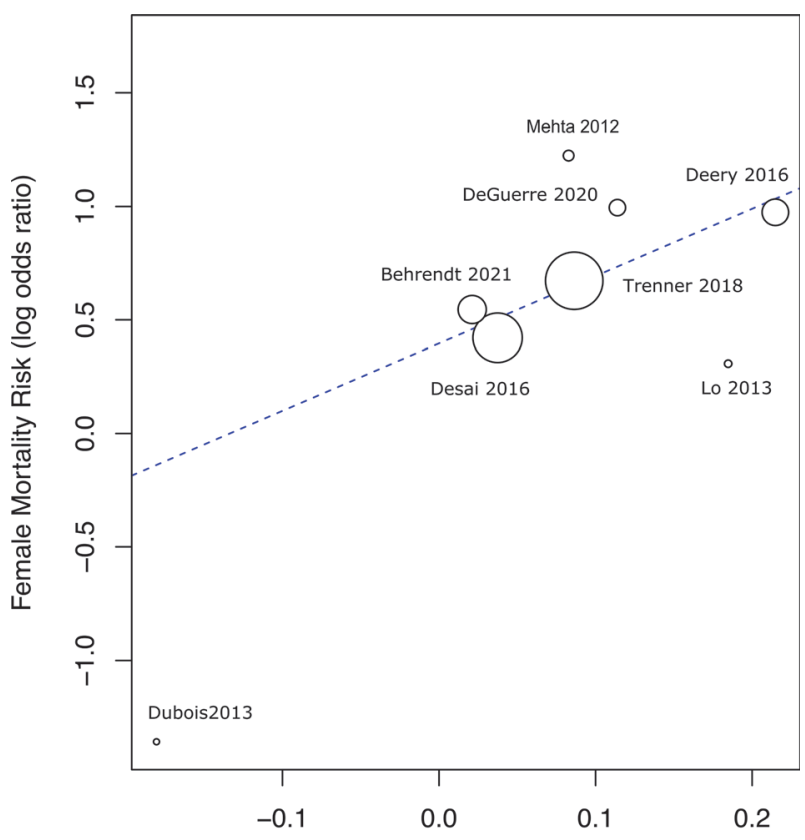

Cardiac Complication (log odds ratio)

\section{Abstract 35 Figure 2}

of AAA (EVAR). (Cardiac: $\mathrm{n}=8, \beta \mathrm{i}=2.96$ (se $=1.27$ ), $\mathrm{p}=0.02$, tau $2=0.00$; renal: $\mathrm{n}=8, \beta \mathrm{i}=2.50 \quad(\mathrm{se}=1.31)$, $\mathrm{p}=0.056$, tau2 $=0.01$ ).

Conflict of Interest None

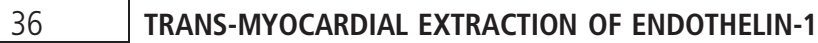 CORRELATES WITH INCREASED MICROVASCULAR RESISTANCE FOLLOWING PERCUTANEOUS CORONARY INTERVENTION}

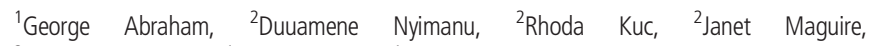
${ }^{2}$ Anthony Davenport, 'Stephen Hoole. ${ }^{1}$ Royal Papworth Hospital NHS Foundation Trust, Cambridge, UK; ${ }^{2}$ University of Cambridge

\subsection{6/heartinl-2021-BCS.36}

Introduction Coronary microvascular dysfunction (CMD) can persist following successful percutaneous coronary intervention (PCI). Endothelin-1 (ET-1) is a potent vasoconstrictor and may be an important mediator of CMD. We sought to assess the trans-myocardial gradient (TMG - coronary sinus minus coronary root levels) of ET-1 and its precursor - Big ET-1 and assess the correlation with pressure-wire indices of CMD: coronary flow reserve (CFR) and index of microvascular resistance (IMR).

Methods Paired blood samples from the aortic root and coronary sinus were collected before and after pressure wire guided PCI from patients with stable angina. Plasma was then analysed using specific enzyme linked immunosorbent assay (ELISA) for quantification of ET-1 and Big ET-1 and correlated with pressure-wire data.

Results Samples were analysed from 66 patients. Both mean ET-1 and Big ET-1 concentrations increased post-PCI in both the aorta (ET-1: $1.0 \pm 0.4 \mathrm{pg} / \mathrm{ml}$ to $1.4 \pm 0.4 \mathrm{pg} / \mathrm{ml}, \mathrm{p}<0.0001$ and Big ET-1: $2.8 \pm 1.3 \mathrm{pg} / \mathrm{ml}$ to $3.4 \pm 1.6 \mathrm{pg} / \mathrm{ml}, \mathrm{p}<0.0001)$ and coronary sinus (ET-1: $1.0 \pm 0.3 \mathrm{pg} / \mathrm{ml}$ to $1.2 \pm 0.3 \mathrm{pg} / \mathrm{ml}$, $\mathrm{p}=0.03$ and Big ET-1: $3.2 \pm 1.7 \mathrm{pg} / \mathrm{ml}$ to $3.8 \pm 1.5 \mathrm{pg} / \mathrm{ml}$,

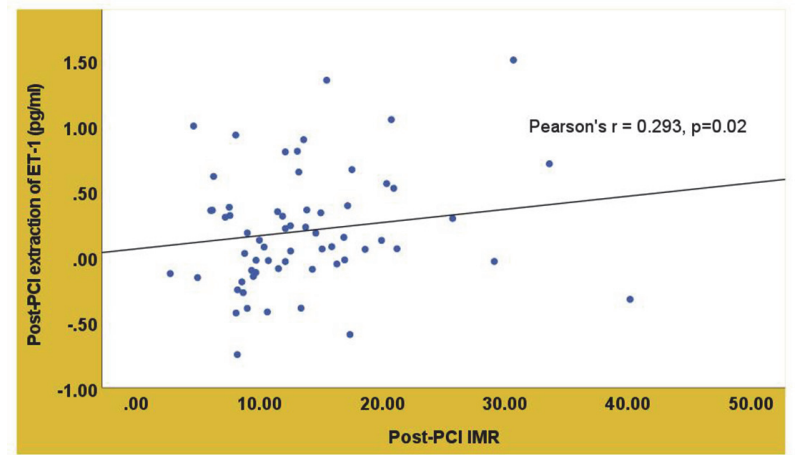

Abstract 36 Figure 1

$\mathrm{p}=0.01)$. TMG extraction of ET-1 increased following PCI: $0.05 \pm 0.25 \mathrm{pg} / \mathrm{ml}$ vs. $-0.20 \pm 0.41 \mathrm{pg} / \mathrm{ml}, \mathrm{p}=0.01$. In contrast, there was TMG release of Big ET-1 before and after PCI: $0.46 \pm 1.26 \mathrm{pg} / \mathrm{ml}$ vs. $0.38 \pm 1.03 \mathrm{pg} / \mathrm{ml}, \mathrm{p}=0.52$ (figure 1). ET1 extraction correlated with IMR post-PCI (Pearson's $\mathrm{r}=$ $0.293, \mathrm{p}=0.02$, figure 2). Patients with $\mathrm{CFR}<2$ post-PCI demonstrated a numerical trend towards higher mean ET-1 extraction than those with preserved CFR post-PCI $(0.30 \pm 0.51 \mathrm{pg} /$ $\mathrm{ml}$ vs. $0.16 \pm 0.42 \mathrm{pg} / \mathrm{ml}, \mathrm{p}=0.31$ ) as did those with criteria for Type $4 \mathrm{a}$ Myocardial Infarction compared with those without $(0.39 \pm 0.57$ vs. $0.15 \pm 0.41, \mathrm{p}=0.11)$.

Conclusions ET-1 and Big ET-1 significantly increase post-PCI. Trans-myocardial extraction of ET-1 increases post-PCI and correlates with post-PCI CMD.

Conflict of Interest none

\section{INCIDENCE AND ONE YEAR OUTCOME OF PERIPROCEDURAL MYOCARDIAL INFARCTION FOLLOWING CARDIAC SURGERY: ARE THE UNIVERSAL DEFINITION AND SCAI CRITERIA FIT FOR PURPOSE?}

${ }^{1} J$ onathan Hinton, ${ }^{2}$ Maclyn Augustine, 'Lavinia Gabara, ${ }^{1}$ Mark Mariathas, 'Rick Allan, ${ }^{1}$ Florina Borca, ${ }^{1}$ Zoe Nicholas, ${ }^{1}$ John Ikwoube, ${ }^{1}$ Neil Gillett, ${ }^{3}$ Chun Shing Kwok, ${ }^{1}$ Paul Cook, ${ }^{2}$ Michael Grocott, ${ }^{3}$ Mamas Mamas, ${ }^{2}$ Nick Curzen. 'University Hospital Southampton, Southampton, UK; ${ }^{2}$ University of Southampton; ${ }^{3}$ University of Keele

\subsection{6/heartjnl-2021-BCS.37}

Introduction The diagnosis and clinical implication of periprocedural myocardial infarction (PPMI) following coronary artery bypass grafting (CABG) is contentious, especially given its importance in the interpretation of trial data. Two accepted definitions of PPMI yield discrepant results. Little is known about the association between the diagnosis of PPMI, using high sensitivity troponin (hs-cTn), and medium term mortality in patients who undergo CABG, either alone or in conjunction with another procedure.

Method Consecutive patients admitted to a cardiothoracic critical care unit (CCCU) over a six month period following open cardiac surgery had hs-cTnI assay performed on admission and every day for forty-eight hours, regardless of whether there was a clinical indication. Patients were categorised as PPMI using both the Universal Definition of MI (UDMI) and Society of Cardiovascular Angiography and Interventions (SCAI) criteria. Comorbidity data, surgical details and clinical progress in CCCU were recorded. One year mortality data were obtained from NHS Digital. 